\title{
The paradoxical association between pulmonary embolism and COPD
}

\author{
Laurent Bertoletti ${ }^{1,2,3}$
}

Affiliations: ${ }^{1}$ Inserm SAINBIOSE, U1059, Dysfonction Vasculaire et Hémostase, Université Jean-Monnet, Saint-Etienne, France. ${ }^{2} \mathrm{CIC} 1408$, Saint-Etienne, France. ${ }^{3}$ Service de Médecine Vasculaire et Thérapeutique, CHU de Saint-Etienne, Saint-Etienne, France.

Correspondence: Laurent Bertoletti, Service de Médecine Vasculaire et Thérapeutique, Hôpital Nord, CHU de St-Etienne, St-Etienne, France. E-mail: laurent.bertolettidchu-st-etienne.fr

@ERSpublications

COPD is associated with an increased risk of PE, but not of VTE recurrence after anticoagulant therapy cessation http://ow.ly/3uio30cAtyd

Cite this article as: Bertoletti L. The paradoxical association between pulmonary embolism and COPD. Eur Respir J 2017; 50: 1700959 [https://doi.org/10.1183/13993003.00959-2017].

Chronic obstructive pulmonary disease (COPD) is recognised as a moderate risk factor (odds ratio between 2 and 9) for venous thromboembolism (VTE) [1], in the same group as cancer or oral contraceptive therapy. However, as opposed to cancer or the pill, this statistical association may represent a clinical dilemma [2], as COPD and the thoracic clinical presentation of VTE (pulmonary embolism, PE) have similar symptoms. The comprehension of the paradoxical association of COPD and VTE, particularly $\mathrm{PE}$, has improved significantly in recent years [3-8].

What do we know?

First, the majority of VTE in COPD patients are provoked [3]. The most frequent trigger is immobilisation for acute medical disease in the past 2 months, such as acute exacerbation of COPD [3]. Secondly, COPD is also a (poor) prognostic factor in VTE patients, with increased risks of both fatal PE [9] and all-cause 30-day mortality [1]. Even if the two previous observations are not surprising, the modified clinical presentation of VTE in COPD patients remains paradoxical and debated. Although deep venous thrombosis (DVT) is usually twice as frequent as PE in the community [10], PE is the most frequent clinical presentation of VTE in patients with $\operatorname{COPD}[3,6,8]$. One explanation could be a diagnostic bias, as presence of respiratory symptoms (due to COPD) may increase the demand for computed tomography and subsequently the number of PE diagnosed. This hypothesis may explain why this modified clinical presentation was also noted in other diseases with respiratory symptoms such as asthma [11], lung fibrosis [12] or chronic heart disease [13]. On the other hand, this potential explanation is totally contradicted by autopsy studies which found that COPD was associated with a high risk of dying from unsuspected PE [14], particularly in patients admitted for acute COPD exacerbation [15]. Hence, specific issues do remain in COPD patients, particularly during COPD exacerbation, such as the frequency of PE (3 or 25\%?) [16] or how to deal with the PE suspicion (Wells or Geneva score?) [17].

Now, just imagine yourself in front of a patient with mild-to-moderate COPD, who was diagnosed 3 months before with PE while he was admitted for increased dyspnoea.

The PE was a first VTE event and unprovoked (no acute medical disease, no surgery, no cancer). This situation is associated with a high risk of recurrence after stopping anticoagulant treatment (20\% at 3 years in the PADIS-PE trial [18]). Regarding the American College of Chest Physicians guidelines [19], the

Received: May 102017 | Accepted: May 122017

Conflict of interest: None declared.

Copyright @ERS 2017 
decision between short or extended therapy (no scheduled stop date) is discussed after at least 3 months, but we have no clear evidence helping us to decide. So, how do we deal with the anticoagulant treatment? Should we consider ourselves (including the patient) lucky that the PE was diagnosed and the patient protected from undiagnosed fatal PE by the anticoagulant therapy? Or should we treat this patient exactly the same as other PE patients, i.e. occulting COPD?

In this issue of the European Respiratory Journal, LE MAO et al. [20] provide solid data on the risk of VTE recurrence in COPD patients in whom anticoagulant therapy is stopped. In this prospective monocentric study evaluating the risk of VTE recurrence after stopping anticoagulant therapy, the authors did not find any difference in terms of global VTE recurrence in patients with COPD versus those without. However, they did demonstrate that PE was the most frequent presentation in case of recurrence, confirming the paradoxical association between COPD and PE. These results were obtained on the whole COPD group, and remain similar in the sensitivity analysis of those with unprovoked VTE. This study has several strengths: to my knowledge, it is the biggest clinical prospective study of patients followed after anticoagulant cessation; the diagnosis of COPD was confirmed by spirometry in most of the cases; the follow-up was extensive (mean of 36.5 months); and all the events were adjudicated by a blinded committee. With regard to its limits, it is a monocentric study involving a team deeply involved both in COPD and VTE care and clinical research. Before being sure of its extrapolability, it should be pointed out that to be included, patients must have stopped their anticoagulant therapy. Hence, we only have the data from patients for whom clinicians felt confident to stop anticoagulant treatment. This potential selection bias was duly recognised in the discussion section [20]. Moreover, the annual incidence of recurrence was elevated: $9.1 \%$ (95\%CI 6.5-12.8\%) for the 136 patients with COPD. We do agree that this rate did not differ significantly from the one found in the 1332 patients without COPD (annual incidence of 7.0\% (95\%CI 6.2-7.9\%)). However, we have to keep in mind that most of these VTE recurrences were PE, and that the case fatality rates differ as widely from VTE recurrence as DVT to VTE recurrence as PE [21].

Hence, although LE MAO et al. [20] confirm that PE is the most frequent clinical presentation of VTE (and then the most frequent form of VTE recurrence), they did not find an increased risk of VTE recurrence (all forms) for which they stopped the anticoagulant therapy, when compared to non-COPD patients.

We now have to answer two crucial questions. First, the annual incidence of recurrence was significantly high, between 7 and 9\% in patients in whom anticoagulant was stopped. The PADIS-PE trial [18] demonstrates that extended vitamin $\mathrm{K}$ antagonists may protect patients from recurrent $\mathrm{PE}$, but increase the risk of major bleeding. Direct oral anticoagulants are as efficient as vitamin $\mathrm{K}$ antagonists in randomised controlled-trials, but with a lower bleeding risk [22]. The recent EINSTEIN-Choice trial [23] opened perspectives for direct oral anticoagulants at reduced-dosage in the extended treatment of VTE. We now have to directly compare full-dosage direct oral anticoagulants to reduced-dosage in the extended treatment of PE, and this trial must include COPD patients. Secondly, the difficulties of PE suspicion in patients admitted for acute COPD exacerbation remain. We hope that the current trials (NCT02035293, NCT02238639) will help clinicians to determine when and how we should manage the PE hypothesis in these patients with acute exacerbation of COPD.

\section{References}

1 Konstantinides S, Torbicki A, Agnelli G, et al. 2014 ESC Guidelines on the diagnosis and management of acute pulmonary embolism. Eur Heart J 2014; 35: 3033-3073.

2 Lippmann M, Fein A. Pulmonary embolism in the patient with chronic obstructive pulmonary disease. A diagnostic dilemma. Chest 1981; 79: 39-42.

3 Bertoletti L, Quenet S, Mismetti P, et al. Clinical presentation and outcome of venous thromboembolism in COPD. Eur Respir J 2012; 39: 862-868.

4 Barba R, Zapatero A, Marco J, et al. Venous thromboembolism in COPD hospitalized patients. J Thromb Thrombolysis 2012; 33: 82-87.

5 Bertoletti L, Quenet S, Laporte S, et al. Pulmonary embolism and 3-month outcomes in 4036 patients with venous thromboembolism and chronic obstructive pulmonary disease: data from the RIETE registry. Respir Res 2013; 14: 75.

6 Børvik T, Brækkan SK, Enga K, et al. COPD and risk of venous thromboembolism and mortality in a general population. Eur Respir J 2016; 47: 473-481.

7 Zöller B, Pirouzifard M, Memon AA, et al. Risk of pulmonary embolism and deep venous thrombosis in patients with asthma: a nationwide case-control study from Sweden. Eur Respir J 2017; 49: 1601014.

8 Kubota Y, London SJ, Cushman M, et al. Lung function, respiratory symptoms and venous thromboembolism risk: the Atherosclerosis Risk in Communities Study. J Thromb Haemost 2016; 14: 2394-2401.

9 Laporte S, Mismetti P, Décousus H, et al. Clinical predictors for fatal pulmonary embolism in 15,520 patients with venous thromboembolism: findings from the Registro Informatizado de la Enfermedad TromboEmbolica venosa (RIETE) Registry. Circulation 2008; 117: 1711-1716.

10 Delluc A, Tromeur C, Le Ven F, et al. Current incidence of venous thromboembolism and comparison with 1998: a community-based study in Western France. Thromb Haemost 2016; 3-10.

11 Majoor CJ, Kamphuisen PW, Zwinderman AH, et al. Risk of deep vein thrombosis and pulmonary embolism in asthma. Eur Respir J 2013; 42: 655-661. 
12 Dalleywater W, Powell HA, Fogarty AW, et al. Venous thromboembolism in people with idiopathic pulmonary fibrosis: a population-based study. Eur Respir J 2014; 44: 1714-1715.

13 Sørensen HT, Horvath-Puho E, Lash TL, et al. Heart disease may be a risk factor for pulmonary embolism without peripheral deep venous thrombosis. Circulation 2011; 124: 1435-1441.

14 Pineda LA, Hathwar VS, Grant BJ. Clinical suspicion of fatal pulmonary embolism. Chest 2001; 120: 791-795.

15 Zvezdin B, Milutinov S, Kojicic M, et al. A postmortem analysis of major causes of early death in patients hospitalized with COPD exacerbation. Chest 2009; 136: 376-380.

16 Aleva FE, Voets LWLM, Simons SO, et al. Prevalence and localization of pulmonary embolism in unexplained acute exacerbations of COPD: a systematic review and meta-analysis. Chest 2017; 151: 544-554.

17 Bertoletti L, Righini M. Adequate use of PE clinical prediction rule in COPD patients. Eur Respir J 2010; 37: 218-219.

18 Couturaud F, Sanchez O, Pernod G, et al. Six months vs extended oral anticoagulation after a first episode of pulmonary embolism. JAMA 2015; 314: 31-40.

19 Kearon C, Akl EA, Ornelas J, et al. Antithrombotic Therapy for VTE Disease: CHEST Guideline and Expert Panel Report. Chest 2016; 149: 315-352.

20 Le Mao R, Tromeur C, Bazire A, et al. Risk of recurrent venous thromboembolism in COPD patients: results from a prospective cohort study. Eur Respir J 2017; 50: 1700094.

21 Carrier M, Le Gal G, Wells PS, et al. Systematic review: case-fatality rates of recurrent venous thromboembolism and major bleeding events among patients treated for venous thromboembolism. Ann Intern Med 2010; 152: 578-589.

22 Bertoletti L, Ollier E, Duvillard C, et al. Direct oral anticoagulants: Current indications and unmet needs in the treatment of venous thromboembolism. Pharmacol Res 2017; 118: 33-42.

23 Weitz JI, Lensing AWA, Prins $\mathrm{MH}$, et al. Rivaroxaban or aspirin for extended treatment of venous thromboembolism. N Engl J Med 2017; 376: 1211-1222. 